\title{
ADDING HIGH POWERED RELATIONS TO LARGE GROUPS
}

\author{
MARC LACKENBY
}

\section{INTRODUCTION}

It is a common theme in group theory that if a group $G$ is quotiented by a sufficiently high power of an element $g \in G$, then properties of $G$ are inherited by the quotient group. A major example of this phenomenon is the theorem of Gromov ([4], see also [2]) asserting that if $G$ is torsion-free and word-hyperbolic, then $G /\left\langle\left\langle g^{n}\right\rangle\right\rangle$ is also word-hyperbolic for all sufficiently big integers $n$. In this paper, we will prove that this phenomenon occurs for the property of being large. Recall that $G$ is large if some finite index subgroup admits a surjective homomorphism onto a non-abelian free group. Large groups have many interesting and useful properties, including super-exponential subgroup growth and infinite virtual first Betti number. They are also particularly important in low-dimensional topology. Our main theorem is the following.

Theorem 1.1. Let $G$ be a finitely generated, large group and let $g_{1}, \ldots, g_{r}$ be a collection of elements of $G$. Then for infinitely many integers $n, G /\left\langle\left\langle g_{1}^{n}, \ldots, g_{r}^{n}\right\rangle\right\rangle$ is also large. Indeed, this is true when $n$ is any sufficiently large multiple of $[G: H]$, where $H$ is any finite index normal subgroup of $G$ that admits a surjective homomorphism onto a non-abelian free group.

There are obvious counter-examples if 'infinitely many integers $n$ ' is replaced by 'all but finitely many integers $n$ ' in the above statement. For example, let $G=\mathbb{Z}_{m} * \mathbb{Z}_{m}$, where $m>2$ and $\mathbb{Z}_{m}$ is the cyclic group of order $m$. Let $g$ be a generator of one of the free factors. Then $G$ is large, but $G /\left\langle\left\langle g^{n}\right\rangle\right\rangle$ is finite cyclic if $n$ and $m$ are coprime. However, when $G$ is free, then we can obtain such a result.

Theorem 1.2. Let $F$ be a finitely generated, free non-abelian group. Let $g_{1}, \ldots, g_{r}$ be a collection of elements of $F$. Then, for all but finitely many integers $n$, the quotient $F /\left\langle\left\langle g_{1}^{n}, \ldots, g_{r}^{n}\right\rangle\right\rangle$ is large.

Theorem 1.1 is in fact a fairly rapid consequence of Theorem 1.2. Like many results about large groups, Theorem 1.2 has a topological proof. One realises $F$ as the fundamental group of a bouquet of circles, and the quotient group $\left.F /\left\langle g_{1}^{n}, \ldots, g_{r}^{n}\right\rangle\right\rangle$ as the fundamental group of the 2-complex $K$ obtained by attaching 2-cells along loops representing $g_{1}^{n}, \ldots, g_{r}^{n}$. The largeness of the quotient $F /\left\langle\left\langle g_{1}^{n}, \ldots, g_{r}^{n}\right\rangle\right\rangle$ is proved by analysing finite-sheeted covering spaces of $K$ corresponding to finite index subgroups of $F /\left\langle\left\langle g_{1}^{n}, \ldots, g_{r}^{n}\right\rangle\right\rangle$. Specifically, the following result of the author (Theorem 3.7 of [6]) will be crucial.

Theorem 1.3. Let $K$ be a finite connected cell complex, and let $A$ and $B$ be subcomplexes such that $K=A \cup B$. Let $p$ be a prime and let $\mathbb{F}_{p}$ be the field of order 
p. Suppose that both of the maps

$$
\begin{aligned}
& H^{1}\left(A ; \mathbb{F}_{p}\right) \rightarrow H^{1}\left(A \cap B ; \mathbb{F}_{p}\right) \\
& H^{1}\left(B ; \mathbb{F}_{p}\right) \rightarrow H^{1}\left(A \cap B ; \mathbb{F}_{p}\right)
\end{aligned}
$$

induced by inclusion are not injections. In the case $p=2$, suppose also that the kernel of at least one of these maps has dimension more than one. Then $\pi_{1}(K)$ is large.

Theorem 1.1 is related to the following result of the author about 3-manifolds (Theorem 3.7 of [5]).

Theorem 1.4. Let $K$ be a non-trivial knot in the 3-sphere, and let $m$ be any integer more than 2. Then, for all sufficiently large integers $n$, the $m n$-fold cyclic cover of $S^{3}$ branched over $K$ has large fundamental group.

Theorem 1.4 does not follow from Theorem 1.1, but Theorem 1.1 is powerful enough to show that the $n$-fold cyclic cover of $S^{3}$ branched over $K$ has large fundamental group, for infinitely many positive integers $n$. The deduction runs as follows. It is a result of Cooper, Long and Reid that $\pi_{1}\left(S^{3}-K\right)$ is large (Theorem 1.3 of [1]). Let $M_{n}$ be the $n$-fold cyclic cover of $S^{3}$ branched over $K$. Then $\pi_{1}\left(M_{n}\right)$ is an index $n$ subgroup of $\pi_{1}\left(S^{3}-K\right) /\left\langle\left\langle\mu^{n}\right\rangle\right\rangle$, where $\mu$ is a representative for a meridian for $K$. Thus, Theorem 1.1 implies that $\pi_{1}\left(M_{n}\right)$ is large for infinitely many $n$.

However, Theorem 1.1 does have some interesting applications to 3-manifold theory, which go beyond Theorem 1.4. In Section 3, we use it to prove that certain 3-manifolds constructed via Dehn surgery have large fundamental group. In fact, we will prove a result (Theorem 3.1) which has the following corollary. (See Section 3 for an explanation of the relevant terminology.)

Theorem 1.5. Let $M$ be a compact orientable 3-manifold with boundary a collection of tori. Suppose that for one collection of slopes $\left(s_{1}, \ldots, s_{r}\right)$, with one $s_{i}$ on each component of $\partial M, M\left(s_{1}, \ldots, s_{r}\right)$ has large fundamental group. Then this is true for infinitely many distinct collections $\left(s_{1}, \ldots, s_{r}\right)$.

Theorem 3.1 provides much more precise information about which collections of slopes $\left(s_{1}, \ldots, s_{r}\right)$ yield 3 -manifolds with large fundamental group.

A result along these lines was proved by Dunfield and Thurston (Theorem 7.3 in [3]). They considered the case where $\partial M$ is a single torus, and where Dehn filling along one slope $r$ yields a Seifert fibre space $\Sigma$ with hyperbolic base orbifold. They also needed to make a hypothesis about how $\pi_{1}(\partial M)$ maps into $\pi_{1}(\Sigma)$. Using the largeness of $\pi_{1}(\Sigma)$, they proved that for infinitely many other slopes $s$ on $\partial M, \pi_{1}(M(s))$ is large. In fact, they showed that this is true when $\Delta(r, s)$ is big enough. Thus, when $M$ has two such slopes $r, \pi_{1}(M(s))$ is large for all but finitely many slopes $s$. A notable example of such an $M$ is the exterior of the figure-eight knot. Although there are some similarities between their approach and ours, there are also some key differences. Our techniques only provide a proof of a weak form of their result. However, their arguments required detailed knowledge of the fundamental groups of Seifert fibre spaces, and so do not readily apply when $M$ has no Seifert fibred Dehn fillings.

\section{Proofs of the Main theorems}

We start by explaining why Theorem 1.1 is a consequence of Theorem 1.2. Let $G$ be a finitely generated group that is large. Let $H$ be a finite index subgroup that 
admits a surjective homomorphism $\psi$ onto a non-abelian free group $F$. Let $g_{1}, \ldots, g_{r}$ be a collection of elements of $G$. Our aim is to show that $G /\left\langle\left\langle g_{1}^{n}, \ldots, g_{r}^{n}\right\rangle\right\rangle$ is large for infinitely many positive integers $n$.

Let $k_{1}, \ldots, k_{s}$ be a set of representatives for the right cosets of $H$ in $G$. Let $m$ be the smallest positive integer such that $k_{j} g_{i}^{m} k_{j}^{-1}$ lies in $H$, for each $i$ and $j$. Note that $m$ is finite, and when $H$ is normal in $G, m$ is the lowest common multiple of the orders of $g_{i} H$ in $G / H$. In particular, $m$ divides the index $[G: H]$ in this case. Let $n$ be any positive integer, and let $G_{m n}=\left\langle\left\langle g_{1}^{m n}, \ldots, g_{r}^{m n}\right\rangle\right\rangle$ be the subgroup of $G$ normally generated by $g_{1}^{m n}, \ldots, g_{r}^{m n}$. Note that this lies in $H$, and is in fact the subgroup of $H$ normally generated by $\left\{k_{j} g_{i}^{m n} k_{j}^{-1}: 1 \leq i \leq r, 1 \leq j \leq s\right\}$. Now, $\left\{\psi\left(k_{j} g_{i}^{m} k_{j}^{-1}\right): 1 \leq i \leq r, 1 \leq j \leq s\right\}$ is a collection of elements of $F$. Hence, by Theorem 1.2,

$$
F /\left\langle\left\langle\psi\left(k_{j} g_{i}^{m n} k_{j}^{-1}\right): 1 \leq i \leq r, 1 \leq j \leq s\right\rangle\right\rangle
$$

is large, for all but finitely many positive integers $n$. This is a homomorphic image of $H / G_{m n}$, which is a finite index subgroup of $G / G_{m n}$. Thus, $G / G_{m n}=$ $G /\left\langle\left\langle g_{1}^{m n}, \ldots, g_{r}^{m n}\right\rangle\right\rangle$ is also large when $n$ is sufficiently big.

Note that this proof also gives some information about the set of values of $n$ for which $G /\left\langle\left\langle g_{1}^{n}, \ldots, g_{r}^{n}\right\rangle\right\rangle$ is large. It includes all sufficiently big multiples of $m$, where $m$ is the positive integer defined above. When $H$ is normal in $G$, we have already seen that $m$ divides $[G: H]$. This proves Theorem 1.1.

We now embark on the proof of Theorem 1.2. Let $F$ be a finitely generated, free non-abelian group. Let $g_{1}, \ldots, g_{r}$ be the given elements of $F$. Our aim is to show that $F /\left\langle\left\langle g_{1}^{n}, \ldots, g_{r}^{n}\right\rangle\right\rangle$ is large for all but finitely many positive integers $n$.

Let $\phi: F \rightarrow \mathbb{Z}$ be projection onto the first free factor, and let $\phi_{n}: F \rightarrow \mathbb{Z}_{n}$ be the composition of $\phi$ with reduction modulo $n$. Realise $F$ as the fundamental group of a bouquet of circles $X$. Let $X_{n}$ be the $n$-fold cyclic covering space of $X$ corresponding to the kernel of $\phi_{n}$. (See Figure 1.)

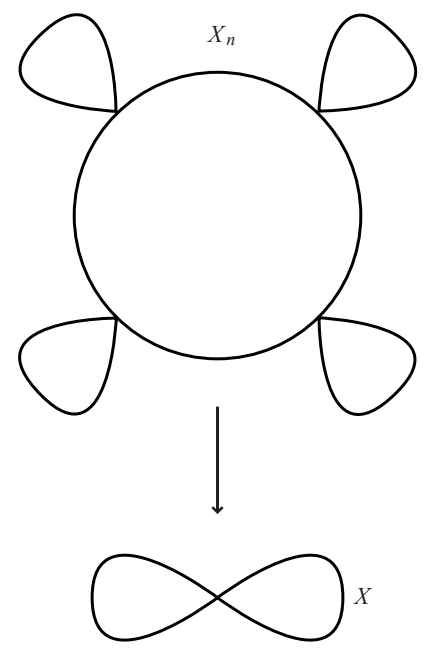

Figure 1 
The elements $g_{1}, \ldots, g_{r}$ can be expressed as reduced words in $F$. They therefore determine loops in $X$, to which we give the same names. Let $K_{n}$ be the 2-complex obtained by attaching 2-cells to $X$ along the words $g_{1}^{n}, \ldots, g_{r}^{n}$. Then the surjective homomorphism $\phi_{n}: F \rightarrow \mathbb{Z}_{n}$ induces a surjective homomorphism $\pi_{1}\left(K_{n}\right) \rightarrow \mathbb{Z}_{n}$. Let $\tilde{K}_{n}$ be the covering space of $K_{n}$ corresponding to the kernel of this latter homomorphism. This is a cell complex obtained by attaching 2-cells to $X_{n}$ along every lift of $g_{i}^{n}$, as $i$ runs from 1 to $r$.

Some 2-cells of $\tilde{K}_{n}$ have the same boundary loops, in the following situation. Suppose that $\phi_{n}\left(g_{i}\right)$ is non-zero in $\mathbb{Z}_{n}$. Then the word $g_{i}$ is an arc in $X_{n}$ that starts and ends at different points. In fact, viewing the vertices of $X_{n}$ as indexed by $\mathbb{Z}_{n}$, the start and end point of $g_{i}$ differ by $\phi_{n}\left(g_{i}\right)$. So, the loop spelling $g_{i}^{n}$ starting at 0 and the loop spelling $g_{i}^{n}$ starting at $\phi_{n}\left(g_{i}\right)$ have exactly the same itineraries, but with different starting points. The same is true for the lift of $g_{i}^{n}$ starting at any element of $\left\langle\phi_{n}\left(g_{i}\right)\right\rangle$, where $\left\langle\phi_{n}\left(g_{i}\right)\right\rangle$ denotes the subgroup of $\mathbb{Z}_{n}$ generated by $\phi_{n}\left(g_{i}\right)$. Thus, we have $\left|\left\langle\phi_{n}\left(g_{i}\right)\right\rangle\right|$ 2-cells all with the same boundary loop. Clearly, we could discard all but one of these 2-cells without changing the fundamental group. Thus, we partition the 2-cells of $\tilde{K}_{n}$ into collections, where two cells belong to the same collection if and only if their attaching loops are lifts of the same $g_{i}^{n}$ and their starting points differ by an element of $\left\langle\phi_{n}\left(g_{i}\right)\right\rangle$. Let $\bar{K}_{n}$ be the 2-complex obtained from $X_{n}$ by attaching just one 2-cell from each collection. This has the same fundamental group as $\tilde{K}_{n}$. Thus, in $\bar{K}_{n}$, the number of 2-cells attached along lifts of $g_{i}^{n}$ is $\left[\mathbb{Z}_{n}:\left\langle\phi_{n}\left(g_{i}\right)\right\rangle\right]$. This is equal to

$$
\begin{cases}\left(n,\left|\phi\left(g_{i}\right)\right|\right) & \text { if } \phi\left(g_{i}\right) \neq 0 \\ n & \text { if } \phi\left(g_{i}\right)=0\end{cases}
$$

where $\left(n,\left|\phi\left(g_{i}\right)\right|\right)$ is the highest common factor of $n$ and $\left|\phi\left(g_{i}\right)\right|$. Note that when $\phi\left(g_{i}\right) \neq 0$, this is at most $\left|\phi\left(g_{i}\right)\right|$.

In the argument that follows, we will treat the elements $g_{i}$ such that $\phi\left(g_{i}\right)=$ 0 in a different manner from those where $\phi\left(g_{i}\right) \neq 0$. Call the former elements $\phi$-trivial, and the latter $\phi$-non-trivial. (See Figures 2 and 3.) If the attaching loop of a 2-cell of $\bar{K}_{n}$ is a lift of $g_{i}^{n}$ and $\phi\left(g_{i}\right)=0$, then we also describe the 2-cell as $\phi$ trivial; otherwise it is $\phi$-non-trivial. Note that, by the above calculation, the number of $\phi$-non-trivial 2-cells in $\bar{K}_{n}$ has an upper bound that is independent of $n$.

Let $p$ be a prime number that divides $n$. The plan is to decompose $\bar{K}_{n}$ into two subsets $A_{n}$ and $B_{n}$ satisfying the conditions in Theorem 1.3. (They will not in fact be subcomplexes of $\bar{K}_{n}$, but this can easily be arranged by subdividing the cell structure of $\bar{K}_{n}$.) Thus, an application of Theorem 1.3 will give that $\pi_{1}\left(\bar{K}_{n}\right)$ is large, and hence so is $F /\left\langle\left\langle g_{1}^{n}, \ldots, g_{r}^{n}\right\rangle\right\rangle$.

We first specify the intersection of $A_{n}$ and $B_{n}$ with $X_{n}$, the 1 -skeleton of $\bar{K}_{n}$. Note that $X_{n}$ is obtained from a circular graph with $n$ edges and $n$ vertices by attaching a collection of loops to each vertex. We may label the $n$ edges of the circle with the integers modulo $n$. Place the dividing line between $A_{n}$ and $B_{n}$ at the midpoints of the edges labelled 0 and $\lceil n / 2\rceil$. Let $P$ be the union of these two points. Specify $A_{n}$ to contain the edges increasing from 1 to $\lceil n / 2\rceil-1$, together with half of the edges labelled 0 and $\lceil n / 2\rceil$. In addition, if any vertex lies in $A_{n}$, then so do the loops that are attached to it. Let $B_{n} \cap X_{n}$ be the remainder of $X_{n}$. (See Figure 4.) 

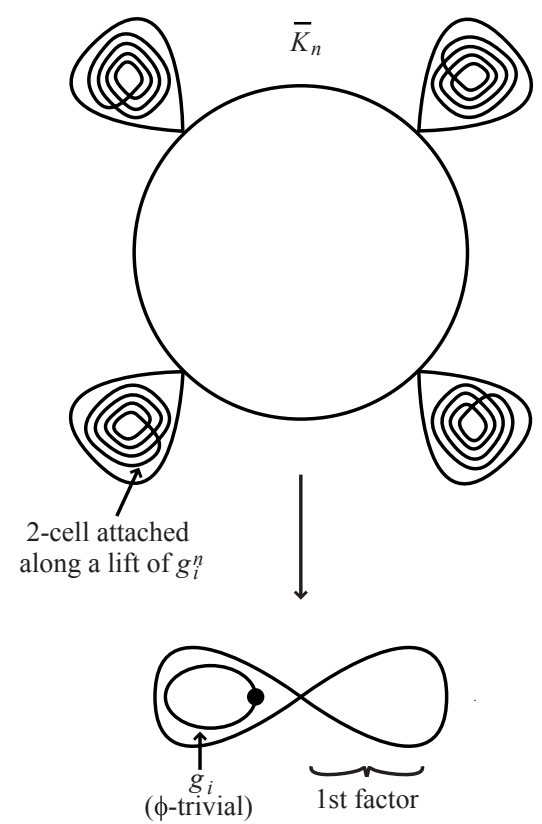

FiguRE 2

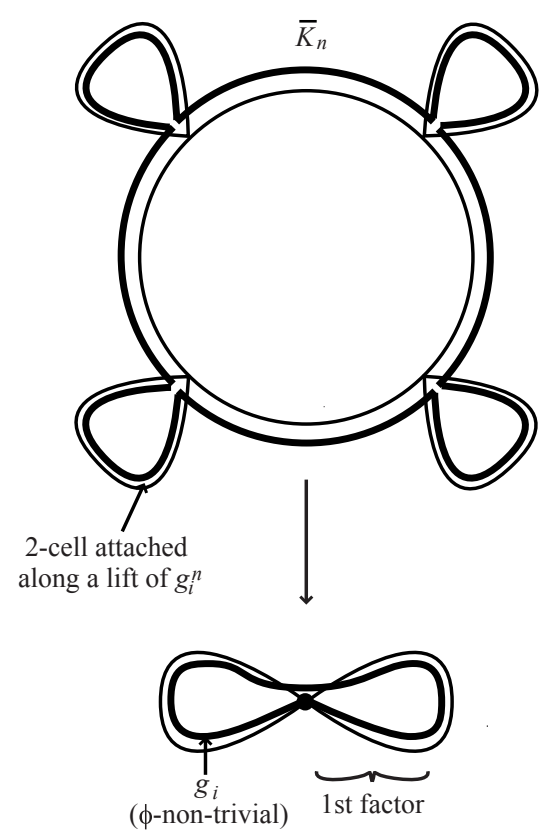

Figure 3 


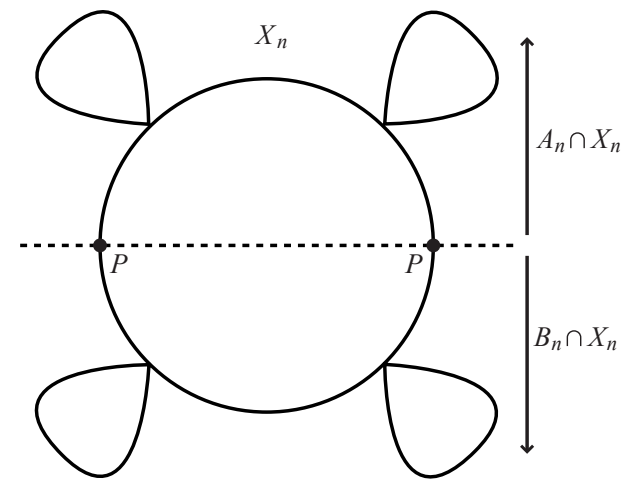

Figure 4

We now define how $A_{n}$ and $B_{n}$ lie within the 2-cells of $\bar{K}_{n}$. If the boundary of such a 2-cell $C$ lies entirely within $A_{n}$ (respectively, $B_{n}$ ), then place that entire 2-cell in $A_{n}$ (respectively, $B_{n}$ ). If not, place a point of $A_{n} \cap B_{n}$ at the centre of $C$. Connect this to each point of $A_{n} \cap B_{n}$ on the boundary of $C$ via a radial arc. Now define $A_{n}$ (respectively, $B_{n}$ ) on $C$, by defining it as the cone on $\partial C \cap A_{n}$ (respectively, $\partial C \cap B_{n}$ ). (See Figure 5.)

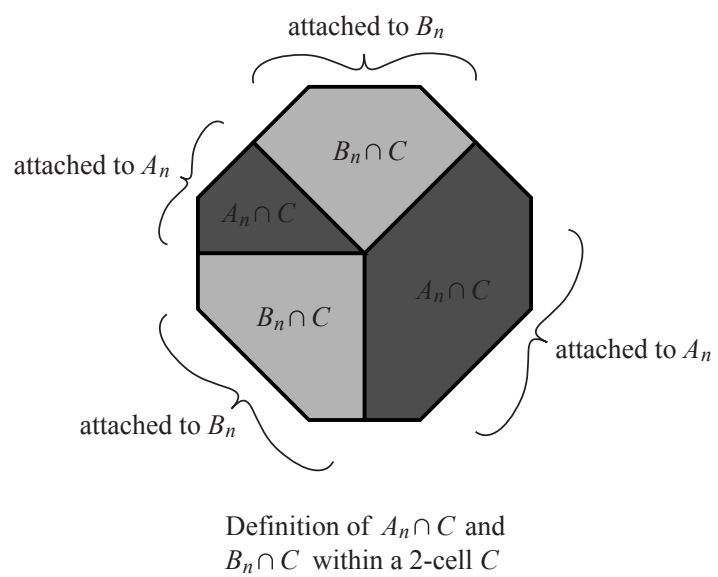

FiguRe 5

In what follows, it will be useful to define a quantity $\Delta_{i}$ associated with each $g_{i}$. Consider the infinite cyclic cover $X_{\infty}$ of $X$ corresponding to the kernel of $\phi$. Now, $\phi$ defines a function from the 0 -skeleton of $X_{\infty}$ onto $\mathbb{Z}$. Extending this linearly over each 1-cell of $X_{\infty}$, this gives a function $X_{\infty} \rightarrow \mathbb{R}$ which we also call $\phi$. Let $\Delta_{i}$ be the difference between the maximum and minimum values of $\phi$ on a lift of $g_{i}$. This is clearly independent of the choice of lift of $g_{i}$. (See Figure 6.)

Claim 1. There is an upper bound, independent of $n$, on the number of times the $\phi$-non-trivial 2-cells of $\bar{K}_{n}$ run over $P$. 


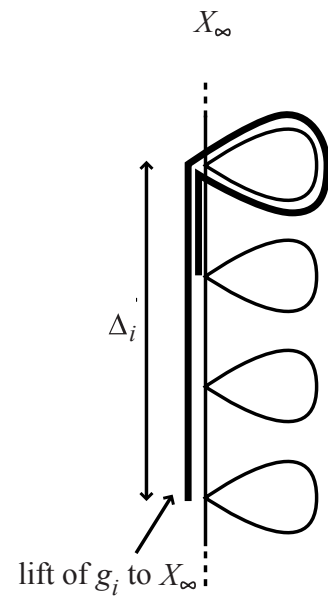

FiguRe 6

Each $\phi$-non-trivial 2-cell of $\bar{K}_{n}$ is attached along a lift of $g_{i}^{n}$, where $\phi\left(g_{i}\right) \neq 0$. Let $j$ be the integer modulo $n$ corresponding to the starting point of this lift. For any integer $k$ between 0 and $n, g_{i}^{k}$ lifts to a sub-arc of this loop, starting at $j$. Its endpoint is $j+k \phi_{n}\left(g_{i}\right)$, modulo $n$. Now, for any given integer modulo $n, j+k \phi_{n}\left(g_{i}\right)$ equals that integer for at most $\left(n,\left|\phi\left(g_{i}\right)\right|\right)$ values of $k$. This is at most $\left|\phi\left(g_{i}\right)\right|$, which is independent of $n$. Hence, there is an upper bound, independent of $n$, on the number of integers $k$ modulo $n$ for which $\left|j+k \phi_{n}\left(g_{i}\right)\right| \leq \Delta_{i}+1$ or $\left|j+k \phi_{n}\left(g_{i}\right)-\lceil n / 2\rceil\right| \leq \Delta_{i}+1$. But when neither of these inequalities is satisfied, the sub-arc of $g_{i}^{n}$ between $g_{i}^{k}$ and $g_{i}^{k+1}$ cannot run over $P$. Thus, there is an upper bound, independent of $n$, on the number of times that this lift of $g_{i}^{n}$ can run over $P$. Since there is a universal upper bound on the number of $\phi$-non-trivial 2-cells in $\bar{K}_{n}$, the claim is proved.

We now specify a graph $\Gamma_{n}$ lying in $\bar{K}_{n}$. It is defined as the union of two sets:

(1) the intersection of $A_{n} \cap B_{n}$ with the $\phi$-non-trivial 2-cells, and

(2) the 1-cells of $X_{n}$ lying in the boundary of each $\phi$-trivial 2-cell that intersects $P$.

Claim 2. There is an upper bound on $d_{p}\left(\Gamma_{n}\right)$, independent of $n$.

Here, $d_{p}(\cdot)$ denotes the dimension of $H_{1}\left(\cdot ; \mathbb{F}_{p}\right)$. Note that $\Gamma_{n}$ has the structure of a graph. Hence, the number of edges of $\Gamma_{n}$ forms an upper bound on $d_{p}\left(\Gamma_{n}\right)$. Its edges come in two types: radial arcs in $\phi$-non-trivial 2-cells running up to $P$, and edges in the boundary of $\phi$-trivial 2-cells that intersect $P$. By Claim 1, there is an upper bound, independent of $n$, on the number of edges of the first type. Each $\phi$-trivial 2-cell runs $n$ times over a lift of $g_{i}$. So, every edge of $\Gamma_{n}$ of the second type lies within a distance $\max _{i} \Delta_{i}$ of $P$. There is therefore a uniform upper bound (independent of $n$ ) on the number of such edges. Thus, the claim is proved.

In the following claim, we show that $d_{p}\left(\bar{K}_{n}\right)$ is bounded below by a linear function of $n$. 
Claim 3. The following inequality holds:

$$
d_{p}\left(\bar{K}_{n}\right) \geq n(d(F)-1)+1-\sum_{i}\left|\phi\left(g_{i}\right)\right| .
$$

Note that $X_{n}$ is a connected graph with $n$ vertices and $n d(F)$ edges. Hence, $d_{p}\left(X_{n}\right)=1-\chi\left(X_{n}\right)=n(d(F)-1)+1$. Now, $\bar{K}_{n}$ is obtained from $X_{n}$ by attaching 2-cells along lifts of $g_{i}^{n}$. When $g_{i}$ is $\phi$-trivial, each corresponding 2-cell runs around a loop $n$ times. So, attaching these does not affect $d_{p}$. (Recall that $p$ divides $n$.) When $g_{i}$ is $\phi$-non-trivial, we attach $\left(n,\left|\phi\left(g_{i}\right)\right|\right)$ 2-cells. This is at most $\left|\phi\left(g_{i}\right)\right|$, and so the claim is proved.

Claim 4. There is a constant $c$, independent of $n$, such that the kernel of

$$
H^{1}\left(\bar{K}_{n} ; \mathbb{F}_{p}\right) \rightarrow H^{1}\left(A_{n} \cap B_{n} ; \mathbb{F}_{p}\right)
$$

has dimension at least $n(d(F)-1)+1-c$.

Here, and throughout the rest of the paper, homomorphisms between cohomology groups will be induced by inclusion, when the context is clear. By Claims 2 and 3, there is a constant $c$, independent of $n$, such that the kernel of

$$
H^{1}\left(\bar{K}_{n} ; \mathbb{F}_{p}\right) \rightarrow H^{1}\left(\Gamma_{n} ; \mathbb{F}_{p}\right)
$$

has dimension at least $n(d(F)-1)+1-c$. We will show that this kernel lies in the kernel of

$$
H^{1}\left(\bar{K}_{n} ; \mathbb{F}_{p}\right) \rightarrow H^{1}\left(A_{n} \cap B_{n} ; \mathbb{F}_{p}\right) .
$$

This will prove the claim. Let $D$ be the union of the $\phi$-trivial 2-cells that intersect $P$, and let $\partial D$ be those 1 -cells that $D$ runs over. The exact sequence associated with the pair $\left(D \cup \Gamma_{n}, \Gamma_{n}\right)$ contains the following terms:

$$
H^{1}\left(D \cup \Gamma_{n}, \Gamma_{n} ; \mathbb{F}_{p}\right) \rightarrow H^{1}\left(D \cup \Gamma_{n} ; \mathbb{F}_{p}\right) \rightarrow H^{1}\left(\Gamma_{n} ; \mathbb{F}_{p}\right) .
$$

By excising $\Gamma_{n}-D$ from $\left(D \cup \Gamma_{n}, \Gamma_{n}\right)$, we see that the first term is isomorphic to $H^{1}\left(D, \partial D ; \mathbb{F}_{p}\right)$, which is trivial. Hence,

$$
H^{1}\left(D \cup \Gamma_{n} ; \mathbb{F}_{p}\right) \rightarrow H^{1}\left(\Gamma_{n} ; \mathbb{F}_{p}\right)
$$

is an injection. So, the kernel of

$$
H^{1}\left(\bar{K}_{n} ; \mathbb{F}_{p}\right) \rightarrow H^{1}\left(\Gamma_{n} ; \mathbb{F}_{p}\right)
$$

equals the kernel of

$$
H^{1}\left(\bar{K}_{n} ; \mathbb{F}_{p}\right) \rightarrow H^{1}\left(D \cup \Gamma_{n} ; \mathbb{F}_{p}\right) .
$$

This clearly lies in the kernel of

$$
H^{1}\left(\bar{K}_{n} ; \mathbb{F}_{p}\right) \rightarrow H^{1}\left(A_{n} \cap B_{n} ; \mathbb{F}_{p}\right)
$$

because this latter map factors through $H^{1}\left(D \cup \Gamma_{n} ; \mathbb{F}_{p}\right)$, as $A_{n} \cap B_{n}$ is a subset of $D \cup \Gamma_{n}$. This proves the claim.

The following claim verifies the key hypothesis of Theorem 1.3.

Claim 5. The kernels of both the following maps

$$
\begin{aligned}
& H^{1}\left(A_{n} ; \mathbb{F}_{p}\right) \rightarrow H^{1}\left(A_{n} \cap B_{n} ; \mathbb{F}_{p}\right) \\
& H^{1}\left(B_{n} ; \mathbb{F}_{p}\right) \rightarrow H^{1}\left(A_{n} \cap B_{n} ; \mathbb{F}_{p}\right)
\end{aligned}
$$

have dimension at least 2 , when $n$ is sufficiently large.

First note that $A_{n} \cap B_{n}$ has at most two components, since every edge of $A_{n} \cap B_{n}$ is incident to $P$, which consists of two points. 
The following is an extract of the Mayer-Vietoris sequence applied to $A_{n}$ and $B_{n}$ :

$$
H^{0}\left(A_{n} \cap B_{n} ; \mathbb{F}_{p}\right) \rightarrow H^{1}\left(\bar{K}_{n} ; \mathbb{F}_{p}\right) \rightarrow H^{1}\left(A_{n} ; \mathbb{F}_{p}\right) \oplus H^{1}\left(B_{n} ; \mathbb{F}_{p}\right) .
$$

Thus, the kernel of

$$
H^{1}\left(\bar{K}_{n} ; \mathbb{F}_{p}\right) \rightarrow H^{1}\left(A_{n} \cap B_{n} ; \mathbb{F}_{p}\right)
$$

maps onto a subspace of the direct sum of the kernels in Claim 5, and the nullity of this mapping is at most the dimension of $H^{0}\left(A_{n} \cap B_{n} ; \mathbb{F}_{p}\right)$, which is at most 2 . We therefore know that the direct sum of the kernels in Claim 5 has dimension at least $n(d(F)-1)-1-c$, by Claim 4 . So, to prove the claim, it suffices to show that each of the kernels in Claim 5 has dimension at most $n(d(F)-1)-3-c$. This will follow from the claim below.

Claim 6. The kernel of each of

$$
\begin{aligned}
& H^{1}\left(A_{n} ; \mathbb{F}_{p}\right) \rightarrow H^{1}\left(A_{n} \cap B_{n} ; \mathbb{F}_{p}\right) \\
& H^{1}\left(B_{n} ; \mathbb{F}_{p}\right) \rightarrow H^{1}\left(A_{n} \cap B_{n} ; \mathbb{F}_{p}\right)
\end{aligned}
$$

has dimension at most $\lceil n / 2\rceil(d(F)-1)+2$.

We focus on the first of these kernels. The argument in the second case is similar. Consider the exact sequence associated with the pair $\left(A_{n}, A_{n} \cap B_{n}\right)$ :

$$
H^{1}\left(A_{n}, A_{n} \cap B_{n} ; \mathbb{F}_{p}\right) \rightarrow H^{1}\left(A_{n} ; \mathbb{F}_{p}\right) \rightarrow H^{1}\left(A_{n} \cap B_{n} ; \mathbb{F}_{p}\right) .
$$

Exactness implies that the kernel of the second map is equal to the image of the first. It therefore suffices to bound the dimension of $H^{1}\left(A_{n}, A_{n} \cap B_{n} ; \mathbb{F}_{p}\right)$ from above. Let $N\left(A_{n} \cap B_{n}\right)$ be a thin regular neighbourhood of $A_{n} \cap B_{n}$. Then $H^{1}\left(A_{n}, A_{n} \cap\right.$ $\left.B_{n} ; \mathbb{F}_{p}\right)$ is isomorphic to $H^{1}\left(A_{n}, N\left(A_{n} \cap B_{n}\right) ; \mathbb{F}_{p}\right)$. By excision, this is isomorphic to $H^{1}\left(A_{n}^{-}, \partial A_{n}^{-}\right)$, where $A_{n}^{-}=A_{n}-\operatorname{int}\left(N\left(A_{n} \cap B_{n}\right)\right)$. (See Figure 7 for an illustration of $A_{n}^{-} \cap C$ and $B_{n}^{-} \cap C$ within a 2-cell $C$.)

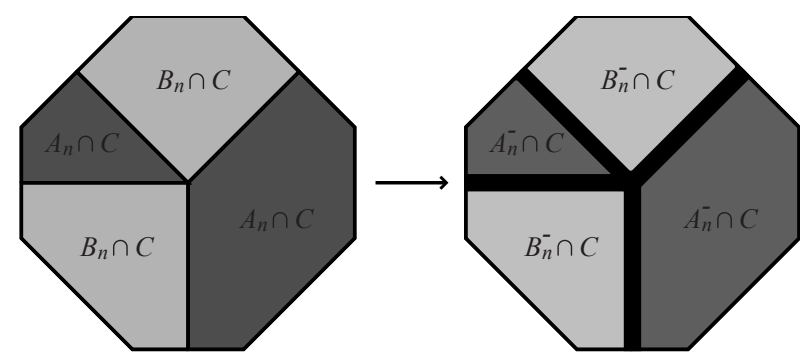

FIGURE 7

The following is an excerpt from the exact sequence for $\left(A_{n}^{-}, \partial A_{n}^{-}\right)$:

$$
H^{0}\left(\partial A_{n}^{-} ; \mathbb{F}_{p}\right) \rightarrow H^{1}\left(A_{n}^{-}, \partial A_{n}^{-} ; \mathbb{F}_{p}\right) \rightarrow H^{1}\left(A_{n}^{-} ; \mathbb{F}_{p}\right) .
$$

This implies that the dimension of $H^{1}\left(A_{n}^{-}, \partial A_{n}^{-} ; \mathbb{F}_{p}\right)$ is at most the sum of the dimensions of $H^{0}\left(\partial A_{n}^{-} ; \mathbb{F}_{p}\right)$ and $H^{1}\left(A_{n}^{-} ; \mathbb{F}_{p}\right)$. Now, $\partial A_{n}^{-}$has at most two components, since each edge of $\partial A_{n}^{-}$is incident to one of two points in $X_{n}$ on the $A_{n}$ side of $P$. Thus, to prove the claim, it suffices to show that

$$
d_{p}\left(A_{n}^{-}\right) \leq\lceil n / 2\rceil(d(F)-1) .
$$


The restriction of $A_{n}^{-}$to any 2-cell $C$ that intersects $P$ is a union of segments of $C$. We may retract these segments onto their intersections with the boundary of $C$, without changing the homotopy type of $A_{n}^{-}$. After this procedure, the 1-skeleton of $A_{n}^{-}$is $X_{n} \cap A_{n}^{-}$. Note that this is connected. Thus,

$$
d_{p}\left(A_{n}^{-}\right) \leq 1-\chi\left(X_{n} \cap A_{n}^{-}\right)=\lceil n / 2\rceil(d(F)-1) .
$$

This proves the claim.

Claim 5 and Theorem 1.3 now give Theorem 1.2.

In the above proof, each of the 2-cells of $\bar{K}_{n}$ was attached along a lift of a loop $g_{i}^{n}$. For the $\phi$-non-trivial 2-cells, it was important that the attaching word was an $n^{\text {th }}$ power. But, for the $\phi$-trivial 2-cells, this was not important. It merely had to be of the form $g_{i}^{m}$, for some $m \geq 2$. Then, working with cohomology modulo $p$, for any prime $p$ dividing $m$, the argument works unchanged. Also, we used a specific homomorphism $\phi: F \rightarrow \mathbb{Z}$, but it is not hard to see that any surjective homomorphism would have worked. Thus, the same argument gives the following variant of Theorem 1.2.

Theorem 2.1. Let $F$ be a finitely generated, free non-abelian group, and let $\phi: F \rightarrow$ $\mathbb{Z}$ be a surjective homomorphism. Let $g_{1}, \ldots, g_{k}$ be a collection of elements of $F$ with trivial image under $\phi$, and let each of $g_{k+1}, \ldots, g_{r}$ have non-trivial image. Then, there is some integer $N$ with the following property. For all $m \geq 2$ and $n \geq N$, the group $F /\left\langle\left\langle g_{1}^{m}, \ldots, g_{k}^{m}, g_{k+1}^{n}, \ldots, g_{r}^{n}\right\rangle\right\rangle$ is large.

\section{Applications to Dehn surgery}

It is a major conjecture that the fundamental group of any finite-volume hyperbolic 3-manifold is large. This is known to be true when the manifold has non-empty boundary by a theorem of Cooper, Long and Reid (Theorem 1.3 of [1]). Many properties that hold for 3-manifolds with toral boundary are also known to be generically true for manifolds obtained by Dehn filling. Thus, 'most' manifolds obtained by Dehn filling a finite-volume hyperbolic 3-manifold should have large fundamental group, but this remains conjectural at present. However, we will show that if it holds for one Dehn filling, then it holds for infinitely many.

We now briefly recall the relevant terminology. Let $M$ be a compact orientable 3 -manifold with boundary a non-empty collection of tori. Then a slope on $\partial M$ is an isotopy class of a simple closed curve which does not bound a disc in $\partial M$. Let $\left(s_{1}, \ldots, s_{r}\right)$ be a collection of slopes on $\partial M$, with at most one $s_{i}$ on each component of $\partial M$. Then $M\left(s_{1}, \ldots, s_{r}\right)$ denotes the manifold obtained by gluing on a collection of $r$ solid tori to $M$ in the following way. We homeomorphically identify the boundary of each solid torus $S^{1} \times D^{2}$ with a boundary component of $M$, so that $S^{1} \times \partial D^{2}$ becomes a curve of slope $s_{i}$. Then $M\left(s_{1}, \ldots, s_{r}\right)$ is said to obtained from $M$ by Dehn filling.

The operation of Dehn surgery is closely related. Now one starts with a compact orientable 3 -manifold $M$, which is possibly closed. Pick a link $L$ in the interior of $M$, and drill out a regular neighbourhood $N(L)$ of $L$, which is a collection of solid tori. Then Dehn filling $M-\operatorname{int}(N(L))$ along every component of $\partial N(L)$ is called performing Dehn surgery on $L$.

For two slopes $s$ and $s^{\prime}$ on a torus, their distance $\Delta\left(s, s^{\prime}\right)$ is the minimal intersection number between representative simple closed curves. 
Theorem 3.1. Let $M$ be a compact orientable 3-manifold with large fundamental group. Let $L$ be a link in $M$, and let $\mu_{1}, \ldots, \mu_{r}$ be a collection of meridians for $L$, one for each component of the link. Then there is a positive integer $N$ with the following property. Let $\left(s_{1}, \ldots, s_{r}\right)$ be a collection of slopes, one on each component of $\partial N(L)$, such that $N$ divides $\Delta\left(\mu_{i}, s_{i}\right)$ for each $i$. Then the manifold obtained by performing Dehn surgery on $L$ via the slopes $\left(s_{1}, \ldots, s_{r}\right)$ has large fundamental group.

Proof. Pick a basepoint for $M-\operatorname{int}(N(L))$, and, for each component of $\partial N(L)$, pick an arc in $M-\operatorname{int}(N(L))$ joining it to the basepoint. Use this to create, for each slope on $\partial N(L)$, a fixed representative element of $\pi_{1}(M-\operatorname{int}(N(L)))$. Let $\lambda_{i}$ be a curve on $\partial M$ such that $\Delta\left(\mu_{i}, \lambda_{i}\right)=1$. Then, in the fundamental group of $M-\operatorname{int}(N(L))$, $s_{i}=\mu_{i}^{p_{i}} \lambda_{i}^{q_{i}}$, where $\left|q_{i}\right|=\Delta\left(\mu_{i}, s_{i}\right)$. Let $M^{\prime}$ be the manifold obtained by Dehn surgery along $L$ via the slopes $\left(s_{1}, \ldots, s_{r}\right)$. Then

$$
\pi_{1}\left(M^{\prime}\right) /\left\langle\left\langle\mu_{1}, \ldots, \mu_{r}\right\rangle\right\rangle \cong \pi_{1}(M) /\left\langle\left\langle\lambda_{1}^{\Delta\left(\mu_{1}, s_{1}\right)}, \ldots, \lambda_{r}^{\Delta\left(\mu_{r}, s_{r}\right)}\right\rangle\right\rangle,
$$

and this has as a quotient

$$
\pi_{1}(M) /\left\langle\left\langle\lambda_{1}^{N}, \ldots, \lambda_{r}^{N}\right\rangle\right\rangle .
$$

Now, for infinitely many $N$, the latter group is large, by Theorem 1.1. Hence, for these values of $N$, so is $\pi_{1}\left(M^{\prime}\right)$.

This has the following corollary.

Theorem 1.5. Let $M$ be a compact orientable 3-manifold with boundary a collection of tori. Suppose that for one collection of slopes $\left(s_{1}, \ldots, s_{r}\right)$, with one $s_{i}$ on each component of $\partial M, M\left(s_{1}, \ldots, s_{r}\right)$ has large fundamental group. Then this is true for infinitely many distinct collections $\left(s_{1}, \ldots, s_{r}\right)$.

Proof. Suppose that for one collection of slopes $\left(\mu_{1}, \ldots, \mu_{r}\right)$, with one $\mu_{i}$ on each component of $\partial M, M\left(\mu_{1}, \ldots, \mu_{r}\right)$ has large fundamental group. The cores of the filled-in solid tori form a link $L$ in $M\left(\mu_{1}, \ldots, \mu_{r}\right)$ with meridians $\mu_{1}, \ldots, \mu_{r}$. Apply Theorem 3.1 to obtain a positive integer $N$, with the following property. For any collection of slopes $\left(s_{1}, \ldots, s_{r}\right)$, with one $s_{i}$ on each component of $\partial M, M\left(s_{1}, \ldots, s_{r}\right)$ has large fundamental group, provided that $\Delta\left(\mu_{i}, s_{i}\right)$ is a multiple of $N$ for each $i$. This clearly holds for infinitely many distinct collections $\left(s_{1}, \ldots, s_{r}\right)$.

\section{References}

[1] D. Cooper, D. Long, and A. Reid, Essential closed surfaces in bounded 3-manifolds, J. Amer. Math. Soc. 10 (1997) 553-563.

[2] T. Delzant, Sous-groupes distingués et quotients des groupes hyperboliques. Duke Math. J. 83 (1996) 661-682.

[3] N. Dunfield and W. Thurston, The virtual Haken conjecture: Experiments and examples, Geom. Topol. 7 (2003) 399-441.

[4] M. Gromov, Hyperbolic groups. Essays in group theory (1987) 75-263 Math. Sci. Res. Inst. Publ.

[5] M. Lackenby, Some 3-manifolds and 3-orbifolds with large fundamental group, Proc. Amer. Math. Soc. (to appear)

[6] Large groups, Property $(\tau)$ and the homology growth of subgroups, Preprint.

Mathematical Institute, University of Oxford, 24-29 St Giles', Oxford OX1 3LB United Kingdom

E-mail address: lackenby@maths.ox.ac.uk 